\title{
Barreiras ao tratamento da hipertensão arterial
}

\author{
Barriers to hypertension treatment
}

Barreras a lo tratamiento de la hipertensión arterial

\section{Maria Vilani Cavalcante Guedes', Thelma Leite de Araujo", Marcos Venícios de Oliveira Lopes", Lucia de Fatima da Silva', Maria Célia de Freitas', Paulo César de Almeida' \\ ' Universidade Estadual do Ceará, Curso de Graduação em Enfermagem, Mestrado Acadêmico em Cuidados Clínicos em Saúde. Fortaleza-CE, Brasil. \\ "Universidade Federal do Ceará, Departamento de Enfermagem. Pesquisador do CNPq. Fortaleza-CE, Brasil.}

Submissão: 30-08-2010 Aprovação: 28-12-2011

\section{RESUMO}

Objetivou-se descrever as barreiras encontradas pelas pessoas portadoras de hipertensão arterial para a não adesão ao tratamento e controle dos níveis de sua pressão arterial. Estudo descritivo e transversal realizado em seis unidades básicas de saúde em Fortaleza-Ceará, Brasil, com 246 pessoas inscritas no Programa de Controle de Hipertensão Arterial há, no mínimo, um ano. Coletaram-se dados com entrevista estruturada e no prontuário eletrônico. Identificaram-se 69 pesquisados com níveis pressóricos normalizados. As principais barreiras apontadas foram: baixas condições financeiras, tratamento contínuo com muitos remédios e prática de atividade física. Concluiu-se que as barreiras ao tratamento anti-hipertensivo compreendem a pessoa adoecida, seu ambiente de vida e acesso aos cuidados de saúde.

Descritores: Enfermagem; Hipertensão; Tratamento.

ABSTRACT
The objective was to describe the barriers faced by people with hypertension for non compliance to treatment and control the levels of blood pressure. This is a transversal and descriptive study which was carried out in 6 basic health units in FortalezaCeará, Brazil. The population consisted of 246 people enrolled in the program to Control Hypertension for at least a year. Data were collected using a structured interview and electronic chart. Of them, 69 showed normal blood pressure levels. The main barriers discovered were: poor financial condition, continuous treatment with many medicines and practice physical activity. It was concluded that barriers to the anti-hypertensive treatment include the sick people, their environment and their access to health care.

Key words: Nursing; Hypertension; Treatment.

\section{RESUMEN}

Se ha objetivado describir los impedimentos encontrados por las personas portadoras de hipertensión arterial para la no adhesión al tratamiento y control de los niveles de su presión arterial. Estudio descriptivo y transversal realizado en seis unidades básicas de salud en Fortaleza-Ceará-Brasil, con 246 personas inscriptas en el Programa de Controle de Hipertensão Arterial hace un año. Se colectaron datos con entrevista estructurada y en el prontuario electrónico. Se identificaron 69 pesquisados con niveles de presión normalizados. Las principales barreras apuntadas fueron: bajas condiciones financieras, tratamiento continuo con muchos remedios y práctica de actividad física. Se concluyó que las barreras al tratamiento anti hipertensión comprenden la persona enferma, su ambiente de vida y acceso a los cuidados de salud.

Palabras clave: Enfermería; Hipertensión; Tratamiento. 


\section{INTRODUÇÃO}

As doenças crônicas se constituem em preocupação em todo o mundo, em virtude da sua morbi-mortalidade, gastos do sistema público de saúde, além das perdas individuais e das dificuldades familiares. De modo geral, o tratamento de qualquer doença crônica não transmissível representa profundo desafio para clientes e profissionais de saúde, sobretudo porque o desaparecimento de sintomas, logo no seu início, induz a pessoa adoecida a acreditar que a doença foi curada.

Esta atitude é observada em portadores de hipertensão arterial, os quais suspendem o tratamento diante do desaparecimento dos sintomas, o que traz sérios prejuízos em decorrência deste comportamento. Isso pode ser apontado como um dos motivos a levar à necessidade de atendimento em serviços de urgência pela ocorrência do aumento da pressão arterial, podendo desencadear sequelas transitórias ou permanentes, ou mesmo causar a morte da pessoa acometida por tal agravo(1).

Como um fator de risco independente e importante para a doença cardiovascular, a hipertensão decorre da relação direta entre morbidade e mortalidade cardiovascular e os valores da pressão arterial. Esta relação é mais evidente para o acidente vascular encefálico (AVE), mas também é presente para doença coronariana (DAC), doença vascular periférica, insuficiência cardíaca e renal. A hipertensão arterial (HA) aumenta o risco de AVE em sete vezes e o infarto do miocárdio em três vezes. Ademais, é a causa mais comum de insuficiência cardíaca, e está associada aos aneurismas em $60 \%$ a $80 \%$ dos $\operatorname{casos}^{(1)}$.

Medidas para manter sob controle os níveis tensionais se constituem nos objetivos do tratamento da HA. No entanto, os clientes tendem a suspender o tratamento não medicamentoso e também o medicamentoso, e, desse modo, contribuem para o elevado índice de morbi-mortalidade por doenças cardiovasculares e cerebrovasculares no Brasil e no mundo. As pessoas com HA justificam este comportamento com argumentos pessoais, econômicos, de relacionamento com os profissionais de saúde que os acompanham e, até mesmo, do ambiente familiar.

No discurso daqueles com HA são comuns algumas afirmações. Entre estas, não faz sentido manter-se em dieta hipossódica, deixar a bebida alcoólica, fazer atividade física todo dia se não têm nenhum sintoma, dando-Ihes a falsa ideia de estarem curados. Isto, porém, vai de encontro ao preconizado pelas mudanças no estilo de vida como componentes fundamentais no controle da doença. A redução do consumo de sal nos alimentos é fundamental, pois além de reduzir os níveis tensionais, determina minimização da mortalidade por acidente vascular cerebral( $^{(2-3)}$, contribui na regressão da hipertrofia ventricular esquerda e na diminuição da excreção urinária do cálcio, colaborando para a prevenção de osteoporose em idosos ${ }^{(4)}$.

No cuidado a esta clientela, sobressai o enfermeiro como o profissional responsável por tal cuidado ao acompanhá-la sistematicamente. Desta maneira, ele poderá colaborar na minimização das barreiras ao tratamento anti-hipertensivo, por meio de orientações, incentivo, acolhimento, escuta qualificada, uso dos recursos disponíveis no serviço para complementar a assistência, valorização de suas dificuldades, medos e objeções ao tratamento.
Em face do exposto, objetivou-se neste estudo descrever as barreiras encontradas pelas pessoas com hipertensão arterial para a não adesão ao tratamento e controle dos níveis de sua pressão arterial, mantendo-os na faixa de normalidade. Desta forma, os resultados alcançados pelo estudo poderão contribuir para o melhor cuidado dispensado pelos profissionais que indicam, prescrevem, acompanham o tratamento e controlam resultados de saúde destas pessoas.

\section{MATERIAL E MÉTODO}

Trata-se de estudo transversal descritivo realizado em seis unidades básicas de saúde da Estratégia Saúde da Família (ESF) em Fortaleza-Ceará-Brasil. Cada unidade contava com quatro equipes e o Programa de Controle de Hipertensão estava implantado há dois anos. Nas seis unidades havia 1.112 pacientes cadastrados no Programa. Destes, 246 participaram do estudo atendendo aos seguintes critérios de inclusão: estar inscrito no Programa de Controle da Hipertensão Arterial há no mínimo um ano, ter comparecido a todas as consultas aprazadas no ano que antecedeu a coleta de dado e não ser portador de qualquer outra doença crônica.

Os dados foram coletados mediante preenchimento de formulário estruturado aplicado por meio de entrevista, cujo roteiro de perguntas dividia-se em duas partes: a primeira para levantar dados sociodemográficos com vistas à caracterização da amostra, e a segunda parte formada por 16 perguntas envolvendo aspectos facilitadores/dificultadores para o seguimento do tratamento anti-hipertensivo. A elaboração do instrumento de coleta de dados, formulário de entrevista, fundamentou-se na definição de barreiras ao tratamento como a avaliação sobre as influências que desencorajam a adoção de ações promotoras de saúde ${ }^{(5)}$.

Além da entrevista com os clientes, utilizou-se um formulário para registro de dados constantes nos prontuários. As informações coletadas dos prontuários foram: resultados das três últimas medições da pressão arterial, peso, altura e tratamento farmacológico prescrito.

Nas entrevistas levantaram-se dados sociodemográficos e sobre alimentação hipossódica e hipolipídica, seja a preparada em domicílio, ou a consumida em restaurantes; a prática regular de atividade física, com duração diária e frequência semanal. Também se investigou a contribuição e compreensão da família e dos amigos para a realização do tratamento. Quanto ao tratamento medicamentoso, questionou-se quantidade, frequência e regularidade no uso. Por fim, indagou-se sobre fatores diversos que facilitam e/ou dificultam o tratamento.

A coleta de dados deu-se no período de setembro a dezembro de 2006 e a escolha dos participantes ocorreu por acessibilidade em salas de espera das unidades de saúde, antes de consultas agendadas para o usuário. Em média, as entrevistas duraram 25 minutos.

Após coletados, os dados foram organizados em uma planilha do Excel. Enquanto os relativos à caracterização da amostra foram descritos por meio de medidas de tendência central e de dispersão das variáveis quantitativas, os relativos às barreiras foram expostos em uma tabela com indicação 
de frequência absoluta. Aplicou-se o teste qui-quadrado para adequação do ajustamento dos resultados, considerando o valor de $50 \%$ das observações como o esperado. Nesse caso, o objetivo era verificar se o percentual de indivíduos que indicaram apresentar determinada barreira ao tratamento era significativamente superior ou inferior a $50 \%$ (maioria ou minoria). Assim, a ocorrência de um valor significativo mostrou quais as barreiras presentes ou ausentes na maioria dos indivíduos avaliados. Adotou-se um nível de significância de 5\%.

Em atendimento ao exigido, a pesquisa foi aprovada pelo Comitê de Ética em Pesquisa do Complexo Universitário Walter Cantídio da Universidade Federal do Ceará, sob o protocolo $n^{\circ} 168 / 06$. Todos os sujeitos foram convidados a participar e aqueles que aceitaram foram esclarecidos sobre objetivos e finalidades do estudo. Após cada aceite, solicitou-se assinatura de um Termo de Consentimento Livre e Esclarecido ${ }^{(6)}$.

\section{RESULTADOS E DISCUSSÃO}

Na sequência, expõem-se descritivamente os resultados de caracterização dos sujeitos e, em uma tabela, são demonstradas as informações relativas às barreiras enfrentadas pela clientela no tratamento da HA.

Como mencionado o grupo estudado compõe-se de 246 sujeitos, dos quais 198 mulheres. A idade variou entre 38 e 89 anos e 97 tinham mais de 60 anos; a renda familiar média foi de até 2,1 salários mínimos e a média de escolaridade foi de 2,1 anos estudados; a maioria morava com outros familiares. Em relação aos níveis de pressão arterial, 69 participantes estavam com pressão arterial consoante parâmetros normais para um portador de hipertensão arterial em uso de anti-hipertensivo, ou seja, < 140x90 mmHg. Deles, 103 foram classificados como portadores de hipertensão arterial estágio I, 68 estágio II e 6 estágio III. Referidas circunstâncias possivelmente se relacionam às barreiras que dificultam o tratamento ${ }^{(7)}$.

Conforme observado, enquanto a monoterapia era praticada por 69 dos entrevistados, os demais faziam uso associado de duas drogas (134), de três drogas (39), e quatro de mais de três drogas. Ainda como observado, 92 usuários disseram tomar todos os medicamentos prescritos, porém a maioria afirmou ser muito desagradável tomar remédio sem apresentar sintomas.

De modo geral, as dificuldades em mudar o estilo de vida e não seguir a prescrição medicamentosa são as duas maiores razões para a pobre aderência das pessoas ao tratamento e controle da $\mathrm{HA}$, apontando para fatores ligados ao paciente, ao provedor de cuidados e ao ambiente terapêutico ${ }^{(5)}$.

Sobressai, particularmente, a exigência de continuidade do tratamento da HA, dificuldades somadas às barreiras para seu seguimento, as quais estão associadas também com a quantidade de drogas prescritas, seus efeitos colaterais, com o nível de confiança e relacionamento entre cliente e profissional e facilidades de acesso aos serviços de saúde.

Portanto, a monoterapia se constitui uma estratégia ideal para iniciar o tratamento da hipertensão arterial e, como tal, foi descrita pelos pesquisados como facilitadora do processo de adesão ao tratamento. Tal fato encontra respaldo na literatura que a considera ideal se a droga for prescrita em dose única ${ }^{(5)}$.

No tocante, ao índice de massa corporal (IMC), os pesquisados foram assim classificados: 62 estavam com IMC normal; 123 estavam com sobrepeso e 61 foram classificados como obesos. No referente, à circunferência abdominal, encontrou-se 86 mulheres com índice $\leq 0,88 \mathrm{~m}$ e 32 homens com índice $<1,02 \mathrm{~m}$, valores considerados normais por sexo ${ }^{(7)}$. De acordo com estes resultados, de certo modo, existe relação entre as barreiras ao tratamento anti-hipertensivo e mudanças no estilo de vida das pessoas com hipertensão arterial. Consoante mencionado, barreiras relacionadas aos hábitos alimentares e ao desenvolvimento regular de uma atividade física contribuem para que outros indicadores se mostrem alterados. Quanto às barreiras ao tratamento e ao controle da pressão arterial enfrentadas pelo grupo estudado, as informações estão expostas na tabela a seguir.

\section{Tabela 1 - Dados relacionados às barreiras ao tratamento e ao controle da pressão arterial segundo os pesquisados. Fortaleza-CE, 2006}

\begin{tabular}{lcccc}
\hline \multicolumn{1}{c}{ Barreiras } & Sim & Não & $\chi^{2}$ & Valor p \\
\hline 1 Faltar condições financeiras & 189 & 57 & 70,82 & $<0,001$ \\
2 Tomar muitos remédios & 182 & 64 & 56,60 & $<0,001$ \\
3 Praticar atividade física & 159 & 87 & 21,07 & $<0,001$ \\
4 Seguir tratamento contínuo & 154 & 92 & 15,62 & $<0,001$ \\
5 Receber ajuda e compreensão da família & 137 & 109 & 3,18 & $>0,05$ \\
6 Tomar remédio mesmo sem sentir sintomas & 126 & 120 & 0,14 & $>0,05$ \\
7 Faltar remédios no posto & 124 & 122 & 0,01 & $>0,05$ \\
8 Comer todo dia com pouco sal & 123 & 123 & 0,00 & $>0,05$ \\
9 Conversar com o médico & 119 & 127 & 0,26 & $>0,05$ \\
10 Comer todo dia com pouca gordura & 116 & 130 & 0,79 & $>0,05$ \\
11 Não usar bebidas alcoólicas & 108 & 138 & 3,65 & $>0,05$ \\
12 Deixar de fumar & 105 & 141 & 5,26 & $<0,025$ \\
13 Reduzir desejo sexual & 98 & 148 & 10,16 & $<0,001$ \\
14 Comer sempre fora de casa & 94 & 152 & 13,67 & $<0,001$ \\
15 Tomar todo dia todos os remédios & 92 & 154 & 15,62 & $<0,001$ \\
16 Conversar com o enfermeiro & 21 & 225 & 169,17 & $<0,001$ \\
\hline
\end{tabular}

Barreiras ao tratamento anti-hipertensivo são todas as dificuldades encontradas ou interpostas pela pessoa com HA para não seguir o tratamento recomendado. Como consta em estudos, as barreiras ao controle da pressão arterial estão em três dimensões: pessoais, emocionais e estruturais ${ }^{(5)}$. Em artigo publicado no American Heart Journal, os autores discutem as barreiras para o controle da hipertensão arterial e apontam a não aderência à prescrição medicamentosa como a maior barreira(5). No estudo em tela, também se percebeu significação estatística $p<0,001$ embora não tenha sido este fator o mais indicado pelos pesquisados.

Como se vê na Tabela 1, somente as quatro primeiras barreiras (faltar condições financeiras, tomar muitos remédios, 
praticar atividade física e seguir tratamento contínuo) estiveram significativamente presentes na maioria dos avaliados. Tais barreiras, ditas da dimensão pessoal, se estabelecem pelas limitações impostas pelo seguimento ideal da terapêutica, ou seja, por mudanças nos hábitos de vida, uso continuado de medicamentos e presença de efeitos colaterais de algumas drogas anti-hipertensivas. Já as emocionais são resultantes do convívio com a doença e suas limitações; e as estruturais se mostram no acesso aos cuidados em serviços de saúde e mesmo a insumos terapêuticos como os medicamentos ${ }^{(5,8)}$. Ademais, idade, sexo, escolaridade e nível socioeconômico são variáveis que devem ser consideradas como barreiras ao tratamento ${ }^{(5,9)}$.

Assim, conforme o presente estudo, as barreiras que dificultam a realização do tratamento anti-hipertensivo e o controle da pressão arterial são de ordem financeira, seguida da necessidade de tomar muitos remédios de modo contínuo e da prática de atividade física, as quais mostraram níveis de significância estatística.

Lembre-se, porém: o tratamento da hipertensão arterial é contínuo e influenciado pelas condições financeiras, envolvendo compra de remédios e alimentos diferenciados. Portanto, o estilo de vida representa outra barreira a ser enfrentada nestas circunstâncias porque as pessoas adoecidas precisam praticar regularmente atividade física e modificar seus hábitos alimentares quanto ao teor de sal e de gordura. A adoção destas mudanças requer persistência e determinação.

Como evidenciado, mudanças no estilo de vida de pessoas com hipertensão arterial se constituem talvez no maior dificultador da adesão ao tratamento, por envolver alimentação, atividade física, abstenção de bebidas alcoólicas e fumo. Na alimentação do brasileiro em geral é comum o uso de sal em quantidade superior ao recomendado ${ }^{(7)}$, assim como de gorduras. Barreiras quanto ao consumo de sal foram encontradas em estudos realizados em Salvador-BA, nos quais 30,2\% da amostra apontaram sua redução como fator que diminui o sabor dos alimentos. Para minorar este efeito, os autores sugerem o uso de limão, vinagre em vez do sal ${ }^{(3)}$, mas, na prática, essa substituição não costuma ocorrer.

Ao se analisar mudanças no estilo de vida no tocante à prática de atividade física, a maioria dos pesquisados o fazem de modo assistemático; portanto, não atendem às recomendações dos consensos nacionais ${ }^{(7)}$. As atividades físicas influenciam na fisiologia de pessoas portadoras de hipertensão arterial, contribuindo para a redução dos níveis de pressão arterial. Desse modo, a não realização regular de atividade física por parte das pessoas entrevistadas é uma barreira ao seu tratamento e controle da HA, além de ser um importante fator de risco para lesões em órgãos-alvo ${ }^{(10)}$. Já os efeitos benéficos do exercício físico produzem bem-estar psicológico e podem ser aproveitados para reduzir o número de medicamentos e de suas doses ${ }^{(11)}$.

No contexto do tratamento da hipertensão arterial, as mudanças no estilo de vida também têm significativa influência. Logo, as pessoas portadoras da doença precisam pensar em como modificar seus hábitos de vida. Urge substituí-los, reduzi-los e incorporar outros mais saudáveis. Assim, as mudanças no estilo de vida de pessoas com doenças crônicas como a hipertensão arterial exigem esforço, perseverança e apoio de familiares, amigos e profissionais de saúde. Com estas iniciativas pode-se contribuir para a modificação ou formação de hábitos saudáveis compatíveis com a nova condição de saúde e de vida, com vistas à adesão total ao tratamento ${ }^{(12)}$.

De modo geral, a prática regular de atividades físicas é uma barreira para as pessoas com hipertensão arterial, em especial às idosas. Mesmo para aquelas que adotam a caminhada como a modalidade escolhida, por ser mais adequada para os menos privilegiados economicamente, há um fator dificultador, a violência urbana, embora o ambiente onde se exercita sejam praças públicas ou áreas do entorno de sua residência.

Outro aspecto a considerar é que a não adesão à terapêutica não medicamentosa contribui para aumento nos valores de parâmetros importantes como o IMC e a circunferência abdominal, assim como sua relação com lesões em órgãos alvo ou mesmo complicações ${ }^{(13)}$.

Sobre os achados do estudo, ressalta-se a ausência de significância estatística nas demais situações questionadas não quer dizer que esses fatores não sejam considerados barreiras importantes ao tratamento da hipertensão arterial. Na verdade, pelos testes aplicados, conforme se pode concluir, a proporção de indivíduos que apontou estes fatores como barreira não difere significativamente de $50 \%$, ou seja, consoante se pode assumir, para a metade dos indivíduos entrevistados, as barreiras descritas nos itens 5 a 11 da Tabela 1 são consideradas fatores dificultadores para o cumprimento do tratamento prescrito.

Com base no teste estatístico usado na análise dos dados, não receber ajuda e compreensão da família, tomar remédio mesmo sem sentir sintomas, faltar remédios no posto, alimentação com pouco sal e pouca gordura, conversar com o médico, e não usar bebida alcoólica podem ser vistos como fatores dificultadores para o tratamento anti-hipertensivo. Todos estes fatores foram mencionados pelos pesquisados como barreira, principalmente em relação à alimentação e à atividade física, pois muitos eram pessoas idosas e necessitavam inclusive de ajuda para caminhar. Para aqueles que costumam fazer refeições fora de casa torna-se difícil o controle de sal e de gorduras. Não poder usar bebidas alcoólicas também se constituiu em barreira para a continuidade do tratamento.

$\mathrm{Na}$ realização de tratamento de doença crônica, a família e o suporte social contribuem para a continuidade regular do tratamento, incentivando, acompanhando ao serviço de saúde, ajudando nas atividades físicas e na tomada de medicamentos ${ }^{(14)}$

Em âmbito mundial, a problemática da baixa adesão ao tratamento da hipertensão arterial é uma preocupação. Em países desenvolvidos, a população tem maior poder aquisitivo, no entanto, encontra-se a adesão em menos de $25 \%$ das pessoas com hipertensão arterial ${ }^{(15)}$. Ao se projetar tal fato para países em desenvolvimento, nos quais a população possui baixo nível socioeconômico e dificuldades, tanto de acesso aos serviços de saúde como de obtenção dos medicamentos prescritos, a não adesão deve ser maior e as consequências serão muito mais graves ${ }^{(3)}$. 


\section{CONCLUSÕES}

Por ser uma doença crônica, ainda incurável, a hipertensão arterial exige várias mudanças no estilo de vida/condutas com vistas ao seu controle. Contudo, estas alterações dependem de decisão individual. Como é notório, fatores de diversas ordens contribuem para a manutenção dessa decisão, enquanto outros são dificultadores. As mudanças no estilo de vida, por exemplo, impõem cuidados com a dieta, controle na ingestão de álcool, cessação do hábito de fumar, manutenção do peso, realização de atividade física regular. Aliado a isso, o seguimento contínuo da prescrição medicamentosa é apontado pela maioria dos pesquisados como inadequado. Tal fato se deve ao desaparecimento de sintomas e, até mesmo, à normalização dos níveis de pressão arterial.

Consoante observado, as barreiras ao tratamento anti-hipertensivo se mostraram em diferentes aspectos como: o tratamento contínuo é cansativo; tomar remédios e fazer dieta sem sentir nenhum sintoma é desagradável. O não uso de bebidas alcoólicas também foi apontado como fator dificultador do seguimento do tratamento.

Ainda como se verificou, as barreiras relacionadas a mudanças no estilo de vida se constituem em fator impeditivo para a adesão ao tratamento da hipertensão arterial. Isto repercute em outros aspectos da vida das pessoas, como a elevação do índice de massa corporal e da circunferência abdominal. Como no grupo avaliado esses parâmetros estavam acima dos valores de normalidade, essas alterações favorecem condições para lesões em órgãos alvo e complicações.

Conforme se acredita, os resultados alcançados neste estudo contribuirão para a compreensão da complexidade do problema da adesão, por profissionais, principalmente os enfermeiros e clientes. Além disso, a sensibilização para o tratamento deve ser uma constante no cotidiano das pessoas envolvidas com a problemática da hipertensão arterial, sejam as pessoas portadoras ou os profissionais que as acompanham.

\section{REFERÊNCIAS}

1. Monteiro MF, Sobral Filho 1. Sales CS, Tamaki EM. Adesão às medidas de controle da hipertensão arterial sistêmica: o comportamento do hipertenso. Cogitare Enferm 2007; 12(2): 157-63.

2. Lima TM, Bucher JSNF, Oliveira Lima JW. A hipertensão arterial sob o olhar de uma população carente: estudo exploratório a partir dos conhecimentos, atitudes e práticas. Cad Saúde Pública 2004; 20(4): 1079-87.

3. Pires CGS, Mussi FC. Crenças em saúde para o controle da hipertensão arterial. Ciênc Saúde Coletiva 2008; 13(Supl 2): 2257-67.

4. Salgado CM, Carvalhaes JTA. Hipertensão arterial na infância. J Pediatr 2003; 79(Supl1): S115-S24.

5. Borzecki AM, Oliveria SA, Berlowitz DR. Barriers to hypertension control. American Heart J 2005: 149 (5); 786-94.

6. Ministério da Saúde (BR), Conselho Nacional de Saúde. Resolução 196, de 10 de outubro de 1996: diretrizes e normas reguladoras de pesquisa envolvendo seres humanos. Brasília (DF); 1996.

7. Sociedade Brasileira de Hipertensão, Sociedade Brasileira de Cardiologia e Sociedade Brasileira de Nefrologia. VI Diretrizes Brasileiras de Hipertensão Arterial. São Paulo; 2010.

8. Pires CGS, Mussi FC. Refletindo sobre pressupostos para cuidar/cuidado na educação em saúde da pessoa hipertensa. Rev Esc Enferm USP 2009; 43(1): 229-36.

9. Pierin AMG, Strelec MAM, Mion Júnior D. O desafio do controle da hipertensão arterial e a adesão ao tratamento. In: Pierin AMG e coordenadora. Hipertensão arterial: uma proposta para o cuidar. Barueri: Manole; 2004. p. 275-89.

10. Souza JA, França ISX. Prevalência de hipertensão arterial em pessoas com mobilidade física prejudicada: implicações para a enfermagem. Rev Bras Enferm 2008; 61(6): 816-21.

11. Medina FL, Lobo FS, Souza DR, Kanegusuku H, Forjaz CLM. Atividade físca: impacto sobre a pressão arterial. Rev Bras Hipertens 2010; 17(2): 103-06.

12. Silva MEDC, Barbosa LDCS, Oliveira ADS, Gouveia MTO, Nunes BMVT, Alves ELM. As representações sociais de mulheres portadoras de hipertensão arterial. Rev Bras Enferm 2008, 61(4): 500-7.

13. Pedrosa RP, Drager LF. Hipertensão arterial sistêmica. Rev Bras Med 2008; 65(12): 74-84.

14. Faé AB, Oliveira ERA, Silva LT, Cadê NV, Mezadri VA. Facilitadores e dificultadores da adesão ao tratamento da hipertensão arterial. Rev Enferm UERJ 2006; 14(1):32-6.

15. Neutel J, Smith DH. Improving patient compliance: a major goal in the management of hypertension. J Clin Hypertens $2003 ;$ 5(2): 127-32. 